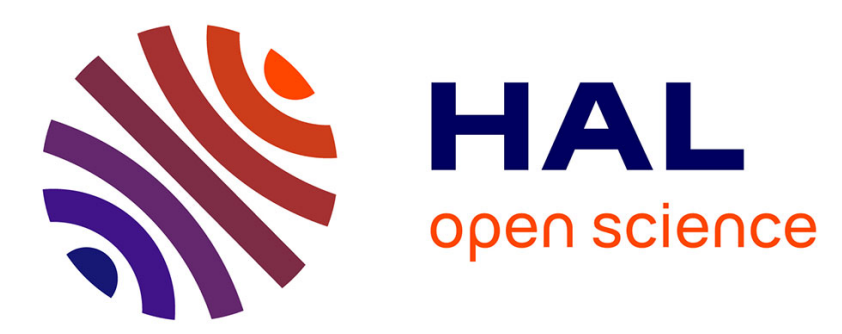

\title{
Energy dispersive X-ray diffraction to identify explosive substances: spectra analysis procedure optimization
}

Charles Crespy, Philippe Duvauchelle, Valerie Kaftandjian, Ferréol Soulez, Pascal Ponard

\section{- To cite this version:}

Charles Crespy, Philippe Duvauchelle, Valerie Kaftandjian, Ferréol Soulez, Pascal Ponard. Energy dispersive X-ray diffraction to identify explosive substances: spectra analysis procedure optimization. Nuclear Instruments and Methods in Physics Research, 2010, 623 (3), pp.1050-1060. hal-00878519

\section{HAL Id: hal-00878519 https://hal.science/hal-00878519}

Submitted on 15 Nov 2013

HAL is a multi-disciplinary open access archive for the deposit and dissemination of scientific research documents, whether they are published or not. The documents may come from teaching and research institutions in France or abroad, or from public or private research centers.
L'archive ouverte pluridisciplinaire HAL, est destinée au dépôt et à la diffusion de documents scientifiques de niveau recherche, publiés ou non, émanant des établissements d'enseignement et de recherche français ou étrangers, des laboratoires publics ou privés. 


\title{
Energy Dispersive X Ray Diffraction to identify Explosive Substances : spectra analysis procedure optimization
}

\author{
Crespy C. ${ }^{1}$, Duvauchelle P. ${ }^{1}$, Kaftandjian V. ${ }^{1}$,Soulez F. ${ }^{1}$, Ponard P. ${ }^{2}$ \\ ${ }^{1}$ CNDRI - Insa Lyon, Université de Lyon, F-69621, Villeurbanne cedex, France \\ ${ }^{2}$ Thales components and subsystems, 2 rue Marcel Dassault 78491, Velizy cedex, France \\ Corresponding author: Charles Crespy \\ email: charles.crespy@,insa-lyon.fr; phillipe.duvauchelle@insa-lyon.fr \\ ad: Insa Lyon, Université de Lyon, CNDRI, F-69621, Villeurbanne cedex, France \\ tel: 0033472437084
}

\section{Abstract:}

To detect the presence of explosives in packages, automated systems are required. Energy dispersive X-ray diffraction (EDXRD) represents a powerful non invasive tool providing information on the atomic structure of samples. In this paper, EDXRD is investigated as a suitable technique for explosive detection and identification. To this end, a database has been constructed, containing measured X-ray diffraction spectra of several explosives and common materials. In order to quantify spectral resolution influence, this procedure is repeated with two different detectors which have different spectral resolution. Using our database, some standard spectrum analysis procedures generally used for this application have been implemented. Regarding to the results, it's possible to conclude on the robustness and the limits of each analysis procedure. The aim of this work is to define a robust and efficient sequence of EDXRD spectra analysis to discriminate explosive substances. Since our explosive substances are crystalline, the first step consists in using characteristic of the spectrum to estimate a crystallinity criterion which allows to remove a large part of common materials. The second step is a more detailed analysis, it consists in using similarity criterion and major peaks location to differentiate explosive from crystalline common materials. The influence of the spectral resolution on the detection is also examined.

Key words: Explosives detection, X-ray diffraction, non destructive testing

\section{Introduction}

Energy dispersive X-ray diffraction (EDXRD) has been successfully used for many years to provide information about the crystalline structure of samples. In this technique, a sample is probed by a 
polychromatic X-ray beam. Photon-matter interactions occur along the incident X-ray beam, in particular, the coherent scattering. The measurement is performed at a fixed scattering angle $(\theta)$ with an energy resolved detector. The spectrum thus obtained is material specific since it is linked to the atomic planar spacing $(d)$ and to the radiation wavelength $(\lambda)$ according to Bragg law: $2 d \sin$ $\theta=n \lambda$ (where $n$ is an integer).

Nowadays, EDXRD is a well established technique developed for a large number of inspection applications (see [Harding'90] and [Luggar'99]). The spectrum analysis procedure is chosen over the major requirements of the application (fast acquisition, accuracy...). Historically, the procedure to recognize an unknown substance from its diffraction spectrum has been developed by Hanawalt in 1936 (see [Langford'96]). It is based on comparison between the main peaks location of the sample spectrum and referenced ones in a paper library. Some particular spectra analysis procedures have been introduced for specific applications. In [Luggar'99b], oil and water ratios are measured in bulk liquid. The analysis spectra procedure consists in performing ratio of the scattering into two energy windows, which provides results with a relative error of $0.6 \%$. [Ballirano'08] reports the first application of EDXRD to the simultaneous structural and compositional characterization of papers. The procedure consists in analyzing the shape of the relevant peak of the crystalline form of cellulose. In [Farquharson'97], authors show that multivariate analysis (MVA) can be a useful technique to estimate components concentration in bones from EDXRD spectra. Principal components are estimated, then calibration bone phantoms are constructed to train the MVA model. The results obtained with measured spectra highlight the influence of exposure time on the accuracy which can reach $3 \%$. For all these studies, the analysis is based on the assumption that the spectrum under testing contains known materials. In the case of luggages inspection, materials number and type are unknown. They can be divided into illicit materials (like drugs, explosives) and common ones (such as sugar, toothpaste...). The identification of illicit materials from their diffraction spectra is usually based on a comparison with a library. One can distinguish different cases : either the library contains only illicit materials spectra, or a complete set of illicit and common materials.

In [Cook'07], X-ray diffraction is proposed as a suitable method to identify illicit drugs in parcels. To this end, spectra of several drugs and possible cutting agents have been used to train a MVA software to identify drug in samples. The technique provides quantitative data of a desired variable (like drug content). In order to reduce the false alarm rate, a database containing the measured spectra of several thousand materials likely found in parcels has to be used. A specific software has been developed to predict EDXRD experimental spectra from powder diffraction profiles 
72 [Cook'09]. The interest is that powder diffraction files are high energy resolution, and thus, the software allows to model different experimental conditions (see [Pani'09]). However, the database must contain a huge number of materials. In [Cook'09b], authors show that MVA predictions based on simulated data are close to MVA predictions based on measured data.

Concerning explosive devices detection inside packages, EDXRD has been examined over the last decade as a suitable tool. In order to reduce the time measurement and to increase the spectral resolution, an experimental device geometry has been established to optimize explosive detection by Luggar and al. (see [Luggar'96] and [Luggar'97]). In [Luggar'98], MVA is performed to detect the presence of explosives. His study demonstrates how MVA may be applied to reduce acquisition time which constitutes one of the main requirement for explosive detection. The limit of this study is the low number of substances used to calibrate the model which leads to a high false alarm rate. In [Malden'00] EDXRD combined with an angular dispersion is examined. It seems to be an efficient way to improve robustness but requires a more complex experimental device. Harding studied the detection of crystalline explosives (see [Harding'99] and [Harding'04]). The data processing takes place in two stages. At first, Wiener filtering is performed to reduce noise in spectra recorded with a low acquisition time, then similarity measure between sample spectra and all library entries is calculated. The author does not disclose the false alarm rate related to this method. In [Harding'06] and [Harding'07] the author focuses on amorphous explosive detection. The main conclusions of the Harding's studies are summed up in [Harding'09].

The present survey concerning substance identification with EDXRD highlights that different kinds of analysis procedures could be implemented successfully. In the present study, a database containing measured EXDRD spectra of several explosives and common materials has been constructed. To construct the database, two kinds of detector, characterized by their spectral resolution, are used. In a second time, different classical analysis procedures are implemented, the robustness and the limit of each methods are examined. Finally we propose a spectra analysis sequence to discriminate explosive substances. The paper is organized as follow: the first section deals with the experimental procedure used to build the library, then the recorded spectra are commented in the second section. Finally the robustness of different analysis procedures are tested in the third section.

\section{EDXRD technique}

\subsection{Experimental arrangement}


109 Schematic representation of experimental device is shown in figure 1. X-ray radiations from a 100

$110 \mathrm{kV}$ tungsten anode $\mathrm{X}$-ray tube operating at $280 \mu \mathrm{A}$ irradiate the sample. The X-ray source has a 10 $111 \mu \mathrm{m}$ focal spot. The scattered photons are detected with detector orientated at $4.28^{\circ}$ from the incident 112 beam. Two types of detectors are used to acquire spectra in this work: CdZnTe or High purity 113 Germanium $(\mathrm{Ge})$. The main difference between both detectors is that $G e$ detector has to be cooled 114 by cryogenic system (it cannot be used in a mobile detection system,) while CdZnTe detector is a 115 room temperature detector. This point leads to a better spectral resolution when Ge detector is used. $116 C d Z n T e$ detector has a FWHM (full width half middle) of $2 \mathrm{keV}$ at $60 \mathrm{keV}$ (see [Verger'07]), while $117 \mathrm{Ge}$ detector has a FWHM of $0.4 \mathrm{keV}$ at $60 \mathrm{keV}$ Another difference between spectra acquired with $118 C d Z n T e$ and $G e$ is the difference between spectral range of both detectors. It can be noticed that the 119 measuring range of Ge detector is from 15 to $100 \mathrm{keV}$ while the measuring range of $C d Z n T e$ 120 detector is from 30 to $100 \mathrm{keV}$. The $C d Z n T e$ detector cut the spectra around $30 \mathrm{keV}$, this limit is not 121 due to the crystal but just an electronic threshold. In the case of luggage inspection, this threshold is 122 out consequence because low energy photons are strongly attenuated thus information under $40 \mathrm{keV}$ 123 is not really useful.

124 Collimation system allows the direct localization of a scatter volume within the sample. The collimator located after the source is a slit of $0.2 \mathrm{~mm}$ width and $8 \mathrm{~cm}$ length. The collimator located in front of the detector is a slit of $0.1 \mathrm{~mm}$ width and $8 \mathrm{~cm}$ length. The scatter angle is a critical parameter which affects the energy resolution, the peaks intensity. It is chosen close to $4^{\circ}$ as a good compromise between angular resolution and main peaks location of explosive substances.

129 Scattering angle is estimated accurately thanks to graphite spectrum measurement performed with $130 \mathrm{Ge}$ detector. The uncertainty of the scatter angle is tor- $0.05^{\circ}$. Finally, the exposure time is set at $131600 \mathrm{~s}$, for each spectra spectra to have a good counting statistic.

\section{$133 \quad 2.2$ EDXRD Spectra}

135 In EDXRD, the spectra acquired depends not only on the sample but also on the experimental set up. An example of an EDXRD acquired with Ge detector spectrum is presented in figure 2.

Figure 2

On figure 2, it can be noted that the spectra is composed of a baseline and peaks. The baseline is caused by the amorphous part of the sample, the incoherent scattered radiation and the spectral shape of X-ray beam. In our configuration, the number of photons whose energy is upper than 90 
$141 \mathrm{keV}$ is too low to provide suitable information in this range of energy. Thus the useful range of 142 energy is from $20 \mathrm{kev}$ to $90 \mathrm{keV}$. The spectral shape of the X-Ray source bias the intensity of the 143 diffraction peaks too: its influence can be removed by normalization to the transmitted spectrum if 144 required.

145 Regarding to the peaks, two kinds of peaks must be distinguished. Some are caused by the 146 characteristic X-ray lines of the X ray tube tungsten target, others are diffraction peaks. Diffraction 147 peaks are the part of the information which is used to identify the sample. The diffraction peaks 148 width are linked to the angular dispersion due to the collimation system and the spectral resolution 149 of the detector. Assuming that diffraction peaks are Gaussian type it can be written:

$$
F W H M_{T}=\left(F W H M_{D}^{2}+F W H M_{C}^{2}\right)^{0.5}(1)
$$

151 Where $F W H M_{T}$ is the total diffraction peak FWHM, $F W H M c$ is the contribution of the collimation 152 system and $F W H M_{D}$ is the contribution of the detector. While $F W H M_{D}$ is known, $F W H M_{C}$ can be 153 estimated experimentally by comparing the width of the diffraction peaks with the width of the 154 fluorescence peaks or numerically by using a Monte Carlo simulation code.

$155 \mathrm{The}_{\mathrm{FWHM}}$ is experimentally estimated: using a CdZnTe detector the FWHM of a diffraction peak 156 is close to $2.4 \mathrm{keV}$, while it's close to $0.95 \mathrm{keV}$ for $\mathrm{Ge}$ detector. These values are of the same order 157 of magnitude than the numerical prediction. In our case it can be noticed that when $C d Z n T e$ detector 158 is used, the detector resolution is the predominant parameter in overall spectral resolution. In the 159 case of the $G e$ detector, the angular resolution due to the collimation system, is the predominant 160 parameter.

161 Concerning the noise it can be reduced by rising the exposure time. In the case of an automated system the exposure time has to be as smaller as possible. As it has been advocated by Harding, the Wiener filter is a suitable tool for noise reduction in EDXRD spectra. The influence of the Wiener filter on $G e$ and $C d Z n T e$ detectors spectra is shown on figure 3.

166 In the following all spectra presented have been filtered.

\section{Database diffraction spectra}

\subsection{Explosives spectra}

172 The spectra of five explosive substances have been recorded: TNT (100\% Tolite), Semtex (91\% 173 RDX), Formex (87\% PETN), PLA NP (89\% PETN) and HCG (98\% RDX). The acquisition time is 174 set to 600 seconds. As an illustration, the filtered spectra of PLA NP and Formex recorded with Ge 
and $C d Z n T e$ detectors are shown in figure 4.

Figure 4

The spectra obtained with the Ge detector show a serie of well resolved peaks. The spectra acquired with the $C d Z n T$ e detector show a series of broader peaks which does not allow to resolve very close peaks. Figure 4 shows the influence of the CdZnTe hardware cut at $40 \mathrm{keV}$. Formex and PLA NP are characterized by the same explosive substance (PETN), this results in two peaks in common around $45 \mathrm{keV}$ which can be seen in Ge spectra.

\subsection{Common material spectra}

In order to evaluate the robustness of the method, spectra of eleven common materials likely to be found in packages have been recorded: $\mathrm{NaCl}$, graphite, PVC, toothpaste, sugar, cotton, soap, aluminum, nickel, copper and coffee. Filtered spectra of PVC and $\mathrm{NaCl}$ recorded with Ge and CdZnTe detectors are shown in figure 5. Unlike explosive spectra, it can be noticed that most of common materials from our library do not show well resolved peaks, but an amorphous trend with broad peaks.

\section{Figure 5}

\subsection{Uncertainty estimation on peaks location}

Since peaks location of a spectra is a capital information about the sample, the uncertainty on the major peak location is estimated. Ten spectra of the same sample successively recorded under similar conditions are compared. The standard deviation on peak location (s) is estimated and used to calculate the uncertainty (2s). This procedure is repeated with three samples ( $\mathrm{NaCl}$, Graphite and Aluminum), for the both detectors, with and without Wiener filtering. The results are shown in figure 6.

\section{Figure 6}

It can be concluded that Wiener filtering is not only a means to remove noise, but also an efficient tool to reduce the uncertainty on peaks location since it allows to reduce significantly the uncertainty with both detectors. After filtering, the uncertainty on peak location for $G e$ detector is less close to $0.2 \mathrm{keV}$ instead of $1.6 \mathrm{keV}$ for $C d Z n T e$ detector. Thus, it is expected to obtain a lower false alarm rate when Ge detector is used.

\section{Spectra analysis procedure}




\subsection{Cristallinity criterion}

212 It can be useful to define a cristallinity criterion to distinguish amorphous materials from

213 crystalline ones. The chosen method is based on the cristallinity degree estimation as performed

214 with angular dispersive $\mathrm{X}$ ray diffraction (see [Cheetham'98]). A smooth curve which connects 215 peak base is estimated using mathematical morphology opening technique (see figure 7).

Figure 7

217 The area above the smooth curve corresponds to the crystalline part while the area under the smooth

218 curve corresponds to the amorphous part. The crystallinity criterion is then estimated as the ratio of 219 upper spectrum area to total diffraction spectrum area. This criterion corresponds to the crystallinity 220 degree used in Angular dispersive X ray diffraction. In the EDXRD case even if the source 221 spectrum shape plays an important role we decide to use the same criterion. The quantitative results 222 of our samples are shown on figure 8. Choosing a crystallinity criterion threshold around 0.2 allows 223 to eliminate a large part of common materials (4 common materials have a cristallinity criterion 224 higher than 0.2) Thus, this crystallinity criterion estimation can be used as a first discriminative 225 step.

Figure 8

\section{4.2. Similarity criterion}

229 As a second step, it has been chosen to identify explosives by calculating a similarity criterion i.e. 230 the cross correlation coefficient.

\section{4.1.1. Acquisition time influence}

232 In order to estimate the robustness of the technique, the influence of acquisition time is investigated.

233 For many substances, six spectra of a same material are acquired with different acquisition times $234(60 \mathrm{~s}, 300 \mathrm{~s}, 420 \mathrm{~s}, 600 \mathrm{~s}, 720 \mathrm{~s}$ and 900s). Then, the cross correlation coefficient is calculated between 235 the spectrum acquired in 900s and the five others. The threshold in terms of cross correlation 236 coefficient is fixed at 0.97 , i.e. if the cross correlation coefficient between two spectra is higher than 237 0.97, the spectra are considered identical. The results for Semtex spectra with and without filtering 238 are shown on figure 9.

Figure 9

240 This example is typical of all investigated cases. The results show that after filtering, spectra 241 acquired with different acquisition time are equivalent. Thus, Wiener filter is a suitable tool to 
242 reduce acquisition time. The results are the same whatever the detector used.

\section{4.1.2. Substance identification}

244 To test the method robustness, a library containing the filtered spectra of each materials is 245 constructed. For a given and unknown material, the cross correlation coefficient is calculated and 246 compared to each library's spectrum in an identification goal. It can be noted that the whole 247 spectrum (amorphous and crystalline part) is used for cross correlation analysis. The results are 248 shown in Table 1.

\section{Table 1}

250 Using the $\mathrm{Ge}$ detector allows to identify a material whatever its crystallinity criterion. Using the $251 C d Z n T e$ detector, the low spectral resolution leads to a partial loss of information. Indeed, although 252 there is no real false alarm case, confusion between some amorphous materials can be noted. For 253 instance, toothpaste can not be distinguished from $P V C$, nor cotton from coffee. The confusion 254 between TNT and Formex is the only confusion between two substances whose cristallinity criterion 255 is upper than 0.2 . Thus, it can be thought that some crystalline common materials could also lead to 256 false alarm.

\subsection{Multivariate Calibration}

260 Detailed description of multivariate calibration procedure can be found in [Cook'07] or 261 [Farquharson'97]. The MVA consists in creating a model in order to link experimental data (X) to 262 quantitative information concerning the sample (Y). As a first step, a linear regression model of the 263 form $\mathbf{Y}=\mathrm{BX}$ is estimated. To this end, a set of calibration spectra is used to train the model. The 264 second step consists in using the model to identify an unknown spectrum. In our case, the 265 experimental $\mathbf{X}$ data are the measured spectra. While, in general, $\mathbf{Y}$ data corresponds to composition 266 of the sample (see [Cook'07] [Luggar'98]). Thus $\mathbf{X}$ is a $N$ dimension vector ( $\mathrm{N}$ corresponds to the number of channels), $\mathbf{Y}$ is a $\mathrm{M}$ dimension vector ( $\mathrm{M}$ corresponds to the number of substances searched for). In practice, it's impossible to perform a well conditioned regression in these conditions, thus $\mathbf{X}$ variables is reduced to a short number of principal components (PCs). Authors show that MVA is a very powerful tool when the spectra of the substances to identify have been used to train the model. Thus, for an unknown package, a database containing several thousands spectra would be required. In our application the presence of explosive is examined, thus $\mathbf{Y}$ is not

273 the sample composition but a threat factor whose value varies from 0 to 1 ( 0 : no explosive in the 274 sample, 1: explosive in the sample). This kind of model is an efficient way to search for a 275 characteristic in explosive spectra which could accurately discriminate them from common 
materials spectra. The model is trained with a set of 84 measured spectra (spectra of the 16 materials acquired with the 5 acquisition time), the number of PCs is 9 . The threshold for detecting the presence of an explosive is fixed to 0.5. Results of the model are shown in figure $\mathbf{1 0 .}$

\section{Figure 10}

It can be concluded that this model leads to one mistake whatever the detector used. In order to check the robustness, the model is tested with spectra from materials which have not been used to train the model. For instance, the model results for water spectra is a false alarm $(\mathbf{Y}(H 2 O)=0.8)$. These findings allow to conclude that it's difficult to identify explosives spectra with a linear model. Thereby, this kind of model is not suitable to discriminate explosive spectra from common materials. The only way to reduce the false alarm rate should be to train the regression model with a complete library containing every common materials spectra which is impossible.

\subsection{Major peaks location analysis}

Another technique inspired from Hanawalt procedure consists in using the major peaks location in order to identify a substance. To perform that a Levenberg-Marquardt minimization algorithm is used to find the sum of Gaussian functions which fit best the spectrum (see figure 11). It's to note that the amorphous part of the spectrum is subtracted before performing the extraction peak procedure.

The standard deviation of the Gaussian function is linked to the detector spectral resolution and the collimation characteristics (see $\$ 2.2$ ). According to Bragg's law, it must be noted that the standard deviation of the Gaussian function is not constant but increases with energy. In first approximation, this point is neglected in the peaks extraction procedure. If a peak corresponds to a characteristic line of the $\mathrm{X}$ ray tungsten, it is not taken into account during the analysis.

Position is given in momentum transfer $(x=\sin (\theta / 2) / \lambda)$ which is a convenient way to express results independently on the diffraction angle. The two main peaks location from $G e$ spectra of each sample are plotted in figure $\mathbf{1 2 .}$

\section{Figure 12}

It can be noted that the spectra of the explosive substances have the main peak located between 1 $\mathrm{nm}^{-1}$ and $1.4 \mathrm{~nm}^{-1}$ (respectively $30 \mathrm{keV}$ and $50 \mathrm{keV}$ when $\theta=4.28^{\circ}$ ). Sugar, soap and graphite also show this characteristic. Using two main peaks allows to reduce the false alarm rate: materials which have the two main peaks located between $1 \mathrm{~nm}^{-1}$ and $1.6 \mathrm{~nm}^{-1}$ (respectively $30 \mathrm{keV}$ and 55 
keV when $\theta=4.28^{\circ}$ ) are explosives only.

310 Regarding now $C d Z n T e$ spectra, the explosive main peaks are located between $1.2 \mathrm{~nm}^{-1}$ and $1.6 \mathrm{~nm}^{-}$

$311^{1}$ (respectively $40 \mathrm{keV}$ and $52 \mathrm{keV}$ when $\theta=4.28^{\circ}$ ). Some spectra of common materials (soap, sugar, 312 graphite) have the same characteristics.

314 The example of graphite is an interesting case. It's known that theoretically the graphite spectrum 315 has just one peak. When a measured peak is broader than the previsions, peaks extraction procedure 316 can lead to the detection of two very close peaks. On figure 3 it can be noted that the main peak of 317 graphite has a non Gaussian shape (asymmetric shape). This asymmetry is related to the height of 318 the collimator which increases the angular aperture with angles above the nominal angle. Moreover, 319 since the graphite peak is located at high energy, it can be though that this point is linked to the 320 variation of standard deviation of Gaussian fit with energy.

321 To sum up, it can be noted that the couple defined by the two main peaks location is material 322 specific whatever the detector used. Moreover all explosives spectra of our library have their main 323 peak located between 1 and $1.6 \mathrm{~nm}^{-1}$. Concerning Ge acquisition, a second peak in this range of 324 energy can be detected. In the future it 's important to check that this observation is valid for every 325 crystalline explosive substances. However, it's possible that others common materials can have 326 these characteristics, probably crystalline organic materials such as drugs.

\subsection{Influence of attenuation on explosive detection}

The aim of this section is to examine if the detection procedure presented above is still valid in the context of our application (when the explosive is in a package). The main problem concerns photon attenuation which depends on their energies and then can significantly modify the diffraction spectrum. Thus, this section focuses on the possibility to identify an explosive spectrum which is modified by a attenuation. The part of the transmitted signal integrated along the wave length can be measured using a scintillator. An experimental procedure has been implemented. It consists in measuring the spectra of an explosive without attenuation, then the experience is repeated when the material is in a package. For example, Figure 14, we can see the presence of explosives (Semtex) into a package containing paper, books and cotton.

341 Six packages containing materials more or less absorbent (cotton, pound, copper ...) are used. Each 342 package is characterized by the percentage of attenuation it causes. The most mitigating package 
343 absorbs $89 \%$ of the total transmitted signal while the least absorbs $15 \%$ of the signal. The figure 15

344 show the influence of attenuation on Semtex spectrum when the transmitted signal is close to $65 \%$.

Figure 15

346 We observe on figure $\mathbf{1 5}$ the difficulty to recognize attenuated from original spectrum. This is due

347 to the fact that attenuation is not spectrally homogeneous, the high energy signal is less attenuated 348 than the signal at low energy. Fortunately, the peaks location is not modified by the attenuation.

349 However, it had be found experimentally that when the transmitted signal is below $30 \%$, the peaks

350 located below $50 \mathrm{keV}$ are no longer detectable. Since the characteristic peaks of explosives are

351 between 30 and $50 \mathrm{keV}$, it is considered that explosive detection is not possible if the transmitted 352 signal is less than $30 \%$.

353 To quantify the influence of attenuation on the cross correlation coefficient identification procedure

354 the cross correlation coefficient is calculated between the spectrum unattenuated and the attenuated 355 one. The results are presented in Figure 16.

Figure 16

357 Note that when the transmitted signal is below $65 \%$, it's no longer possible to recognize the 358 spectrum of Semtex using the correlation coefficient. Since attenuation can damage the spectra 359 shape of a sample, the thresholds of all detection procedures have to be redefined as a function of 360 attenuation.

\section{Spectra analysis sequence}

364 Taking into account the conclusions of the section 4, an algorithm dedicated to automatic detection 365 has been implemented. The principle is illustrated in figure 17. The two entries of the algorithm is 366 sample spectra and the integrated attenuation.

Figure 17

368 First, the integrated attenuation is used to define the detection reliability. This parameter influence 369 the detection thresholds of the detection procedure. If the transmitted signal is less than $30 \%$ of the 370 initial signal, it is considered that the identification is not possible.

371 If the detection reliability is sufficient, the sample spectrum is analyzed. The sample spectrum is 372 first filtered, then the crystallinity criterion is calculated. If the spectrum of the sample is of 373 crystalline type (crystallinity criterion $>0.2$ ), the cross correlation coefficient is used to compare the 374 sample spectra with the explosive spectra of our library. Finally, in case of non detection, peaks 375 location of the sample are compared with those of explosives taking into account the uncertainty. 
376 It's to note that the whole spectrum (amorphous and crystalline part) is used in cross correlation 377 analysis, while only the crystalline part is used for peak location analysis. In case of using $C d Z n T e$ 378 spectra, only the two main peaks are used for identification while when Ge detector is used it's possible to compare a larger number of peaks in order to reduce the false alarm rate. This step is important to detect explosive substance in inhomogeneous voxel. The three step detection procedure allow to increase the robustness. Moreover, the graphic user interface (GUI) allows a direct visual comparison between sample spectra and those of the library. To illustrate, Figure 18 shows a screen-shot of the graphic user interface.

Figure 18

\section{Conclusion}

In this study, explosive detection using EDXRD has been investigated using a library containing five explosives and eleven common materials. As a first step it was chosen to study crystalline explosives. Thus a crystallinity criterion calculation is proposed in order to discriminate them from common materials. This step allow to discriminate easily many common materials from explosive ones. To perform a more accurate discrimination three classical spectra analysis procedures have been implemented: similarity criterion calculation, MVA and main peaks location. It has been shown that the use of MVA requires to build a complete library containing not only explosives spectra but also every common materials spectra. That's why this technique is considered as not suitable for package inspection. On another hand, explosive spectra show some discriminant characteristics in term of peaks location and cross correlation coefficient. Whatever the analysis procedure, the Ge spectra are more suitable for discrimination than CdZnTe. Thus CdZnTe detector can be applied as a complementary technique in a portable system in order to reduce the false alarm rate.

400 Following this study, the library will be completed with other explosive spectra, and the detection 401 technique will be tested with more crystalline common materials in order to estimate accurately the 402 false alarm rate. Moreover, to improve the discrimination rate with $C d Z n T e$ detector it's envisaged 403 to decrease the scattering angle at $3^{\circ}$ and redefine the collimation system.

\section{Acknowledgements}

406 This work was conducted in the frame of the SPIDERS project, supported by the French National 407 research agency. Specifically, we wish to thank the CEA LETI for having lent the CdZnTe detector 408 and ETBS for having supplied the explosive substances. 


\section{References}

411 [Ballirano'08] Ballirano P., Caracciolo G., Sadun C., Caminti R., The use of energy X -ray

412 diffraction (EDXD) for the investigation of th structural and compositional features of old and 413 modern papers, Microchemical Journal 88 (2008) pp.107-112

414 [Cheetham'98] Cheetham N.W.H., Tao L., Variation in crystalline type with amylose content in 415 maize starch granules: an X-ray powder diffraction study, Carbohydrate Polymers 36 (1998), 416 pp.277-284

417 [Cook'07] Cook E., Fong R., Horrock J.A, Wilkinson D., Speller R.D, Energy dispersive X ray 418 diffraction as a means to identify illicit materials A preliminary optimisation study, Appl. Radiat. 419 Isot Vol. 48, No.2, ( 2007) pp. 215-224

420 [Cook'09] Cook E., Griffits J.A., Koutalonis M., Gent C., Pani S., Horrock J.A, George L., 421 Hardwick S., Speller R.D, Illicit drug detection using energy dispersive x-ray diffraction Proc. Of 422 SPIE vol. 7310, 73100I (2009)

423 [Cook'09b] Cook E., Pani S , George L, Hardwick S., Horrock J.A,., Speller R.D, Multivariate 424 data analysis for drug identification using energy-dispersive x-ray diffraction, IEEE transactions on 425 nuclear science Vol. 56, No.3 (2009) pp. 1459-1460

426 [Farquharson'97] Farquharson M.J., Luggar R.D., Speller R.D., Multivariate calibration for 427 quantitative Analysis of EDXRD spectra from a bone phantom, Appl. Radiat Isot. Vol 48 No.8 428 (1997) pp. 1075-1082

429 [Harding'90] Harding G., Newton M., Kosanetzhy J., Energy dispersive X ray diffraction 430 tomography, Phys. Med. Biol. Vol. 35 No 1. (1990) pp, 33-41

431 [Harding'99] Harding G., Schreiber B., Coherent X-ray scatter imaging and its applcations in 432 biomedical science and industry, Radiation Physics and Chemistry 56 (1999) pp.229-245

433 [Harding'04] Harding G., X ray scatter tomography for explosives detection, Radiation Physics 434 and Chemistry, 71, (2004) pp. 869-881

435 [Hardin'06] Harding G., Effective density and atomic number determined from diffraction profiles, Proc. of SPIE Vol. 6319, 631910 (2006)

437 [Harding'07] Harding G., Delfs J.,Liquids identification with X ray diffraction, Proc. of SPIE Vol. 438 6707, 67070T-1 (2007) 
440 [Harding'09] Harding G., X-ray diffraction imaging a multi generational perspective, Applied 441 radiation and isotropes 67 (2009), pp. 287-295

442 [Langford'96] Langford I., Louër D., Powder diffraction, Rep. Prog. Phys. 59 (1996) p.131-254

443 [Luggar'96] Luggar R.D., Horrock J.A., Speller R.D., Lacey R.J., Determination of geometric

444 blurring o an energy dispersive $\mathrm{X}$ ray diffraction (EXRD) system and its use in the simulation of 445 experiments derived diffraction profiles, Nuclear Instruments and Methods in Physics Research A 446383 (1996) pp. 610-618

447 [Luggar'97] Luggar R.D., Horrock J.A., Speller R.D., Lacey R.J., Low angle X ray scatter for 448 explosives detection: a geometry optimization, Appl. Radiat. Isot Vol. 48, No.2, (1997 ) pp. 215-224

449 [Luggar'98] Luggar R.D., Farquharson M.J., Horrocks J.A., Lacey R.J., Multivariate analysis of 450 statiscally poor EDXRD spectra for the detection of concealed explosives, X-ray spectrometry 451 vol,27 (1998) pp. 87-94

452 [Luggar'99] Luggar R.D., Gilboy W.B., Recent developments in industrial applications of elastic 453 scatter X-ray inspection, Radiation Physics and Chemistry 56 (1999) pp. 213-227

454 [Luggar'99b] Luggar R.D., Key M.J., Morton E.J., Gilboy W.B., Energy dispersive X ray scatter 455 for measurement of oil/water ratios, Nuclear Instruments and Methods in Physics Research A 422 456 (1999) pp.938-941

457 [Malden'00] Malden C.H., Speller R.D., A Cd Zn TE array dor the detection of explosives on 458 baggage by energy dispersive $\mathrm{X}$ ray diffraction signatures at multiple scatter angles, Nuclear 459 Instruments and Methods in Physics Research A449, (2000) pp. 408-415

460 [Pani'09] Pani S, Cook E., Horrock J., George L, Hardwick S, Speller R., Modeling an energy461 dispersive x-ray diffraction system for drug detection, IEEE transactions on nuclear science Vol. $462 \quad 56$, No.3 (2009) pp. 1238-1241

463 [Verger'07] Verger L., Gros d'Aillon E., Monnet O., Montémont G., Pelliciari B., New trends in g 464 ray imagining with CdEnTe/CdTe at CEA- Leti, Nuclear Instruments and Methods in Physics 465 Research A571, (2007) pp. 33-43 


\section{Figures caption:}

467 figure 1: schematic representation of the experimental setup

468 figure 2: Example of a diffraction spectrum acquired with $\mathrm{Ge}$ detector

469 figure 3: visualization of Wiener filter influence on measured spectra with $C d Z n T e$ (a) and with Ge 470 (b)

471 figure 4: examples of explosive spectra measured with a $G e$ detector (left) and a $C d Z n T e$ detector 472 (right)

473 figure 5: examples of common materials spectra measured with a Ge detector and a $C d Z n T e$ 474 detector

475 figure 6: peak location uncertainty

476 figure 7: estimation of amorphous area (gray zone) with morphological opening of the global 477 spectrum (on the left for the PLA NP and on the right for PVC)

478 figure 8: cristallinity criterion of our samples

479 figure 9: cross correlation coefficient as a function of exposure time for Semtex and the 2 studied 480 detectors

481 figure 10: MVA results for $C d Z n T e$ and Ge spectra

482 figure 11: examples of peaks extraction in $T N T$ spectra acquired with $C d Z n T e$ detector (a) and Ge 483 detector (b)

484 figure 12: two main peaks location of $G e$ spectra $\theta=4.28^{\circ}$

485 figure 13: two main peaks location of $C d Z n T e$ spectra $\theta=4.28^{\circ}$

486 figure14: visualization of a package containing an explosive substance

487 figure 15: visualization of the influence of attenuation on Semtex spectrum acquired with Ge 488 detector when the transmitted signal is close to $65 \%$

489 figure 16: influence of the attenuation on the substance identification with cross correlation

490 coefficient

491 figure 17: detection algorithm principle

492 figure 18: illustration of the interface of the detection algorithm

\section{Table caption:}

495 Table 1: substance identification using cross correlation coefficient 


\section{Table:}

497

\begin{tabular}{|c|c|c|}
\hline Material Tested & Germanium detector & CdZnTe detector \\
\hline Aluminum & Aluminum & Aluminum \\
\hline Coffee & Coffee & $\begin{array}{l}\text { Coffee } \\
\text { Cotton }\end{array}$ \\
\hline Copper & Copper & Copper \\
\hline Toothpaste & Toothpaste & $\begin{array}{l}\text { Toothpaste } \\
\boldsymbol{P} \boldsymbol{V} \boldsymbol{C}\end{array}$ \\
\hline Graphite & Graphite & Graphite \\
\hline $\mathrm{NaCl}$ & $\mathrm{NaCl}$ & $\mathrm{NaCl}$ \\
\hline Nickel & Nickel & Nickel \\
\hline$P V C$ & $\mathrm{PVC}$ & $\begin{array}{c}\text { PVC } \\
\text { Toothpaste }\end{array}$ \\
\hline Sugar & Sugar & Sugar \\
\hline Soap & Soap & Soap \\
\hline Cotton & Cotton & $\begin{array}{l}\text { Cotton } \\
\text { Coffee }\end{array}$ \\
\hline Semtex & Semtex & Semtex \\
\hline Formex & Formex & $\begin{array}{c}\text { Formex } \\
\boldsymbol{T N T}\end{array}$ \\
\hline$P L A N P$ & PLA NP & PLA NP \\
\hline$H C G$ & HCG & HCG \\
\hline$T N T$ & $\mathrm{TNT}$ & $\begin{array}{c}\mathrm{TNT} \\
\text { Formex }\end{array}$ \\
\hline
\end{tabular}

